\title{
A Proactive Solution, using Wearable and Mobile Applications, for Closing the Gap between the Rehabilitation Team and Cardiac Patients
}

\author{
Remus A. Dobrican \\ University of Luxembourg \\ Computer Science and Communication Research Unit \\ Luxembourg, Grand-Duchy of Luxembourg \\ Email: remus.dobrican@uni.lu
}

\author{
Denis Zampunieris \\ University of Luxembourg \\ Computer Science and Communication Research Unit \\ Luxembourg, Grand-Duchy of Luxembourg \\ Email: denis.zampunieris@uni.lu
}

\begin{abstract}
Exercise training (ET) is one of the decisive and crucial factors for reducing and preventing unexpected cardiac events. The aim of this work is to implement a proactive eHealth system that will allow patients, following a Cardiac Rehabilitation (CR) program outside the hospital, to exercise safely, according to their recommended training zones. The eHealth system includes a smartwatch application, a smartphone application and several server-side applications, working on predefined personalised scenarios, which are alerting, guiding and supporting the patients. The heart rate (HR) of patients is continuously measured, recorded and analysed during ET with the help of wearable devices. While training, patients benefit from multiple levels of feedback. Communities of patients are created for stimulating and motivating patients to perform according to their CR program. Opposed to traditional home-based CR eHealth applications, profiles and training zones are created and handled dynamically for each patient.

Keywords-e-Health, wearable application, smartphone application, cardiac rehabilitation, proactive systems, personalized scenarios, automated patient monitoring.
\end{abstract}

\section{INTRODUCTION}

Even though the rate of death caused by heart diseases has decreased in the past years, the risks of heart failure are still very high, heart diseases being accountable for almost one third of the worldwide deceases [1]. People that suffered from a heart attack or have a heart disease/condition need to reduce the probability of a potential future fatal heart event and one of the main ways of doing this is Cardiac Rehabilitation.

\section{A. Cardiac Rehabilitation}

Cardiac Rehabilitation has many definitions [2][3], being seen as a set of key activities, including physical, social and mental factors, which slow, or even reverse, the evolution of the patient's disease or condition [4]. The benefits of CR include reducing the rate of mortality and unplanned hospital visits [5], lowering blood pressure and stress [6], improving the patient's exercise capacity and improves the overall quality of life [7].

Despite the major benefits of $\mathrm{CR}$, the rate of participation in $\mathrm{CR}$ programs remains quite low [8]. To address this problem, new strategies for keeping the patients motivated and engaged in the CR programs need to be created. The reasons for the low attendance in CR programs differ from one phase to another. The process of cardiac rehabilitation is traditionally divided into four phases. Phase- 1 starts at the hospital, phase- 2 and phase- 3 continue at the patient's home with periodical visits to the hospital, and phase- 4 is the long term maintenance process for staying healthy.

This study focuses on phase- 2 and phase- 3 of the CR program, where the patients have to perform multiple sets of exercises in an environment that does not have medical equipment nor specialists to guide them, which is possibly less safe. Studies indicate that patients in phase-2 of CR are most vulnerable because they tend to do intensive exercises, if not guided correctly, that can have devastating effects [9] 【10].

\section{B. Wearable devices}

Recently, major advances in wearable technology, such as sensor technology, communication technology and data analysis methods [11], give the possibility to asses the physiological health of patients, by collecting data in a non-invasive and non-obtrusive way [12]. Wearable devices were successfully used as monitoring and alerting systems in case of highrisk cardiac patients [13], of analysing Parkinson patient's movement for detecting and preventing freezing of gait [14] and for estimations of heart and breathing rates from wrist motions [15]. The newest generation of smartwatches is now capable of measuring the HR very accurately with the help of photoplethysmography (PPG) technology [16]. The HR is a crucial health parameter measured in most of the CR programs.

\section{Contribution}

Our work is aiming at proposing a proactive IT system capable of performing automated patient monitoring, of providing patients with real-time expert feedback, of alerting the patients in case they perform exercises too fast or too intensive, of alerting the medical experts in case an emergency is detected, 
of integrating patients into communities of cardiac patients that are also training at home and of tracking and comparing all the sessions of all the patients. The IT system is composed of an application for smartwatches, an application for mobile phones and an application for the central server, and it is based on Proactive Computing [17], a recent computing paradigm for IT systems that works on behalf of the users and for the users [18]. More specifically, a set of Proactive Scenarios [19] is developed and integrated into the mobile phones and into the server in order to include the knowledge of the medical experts and to react to different events such as, for example, the sudden increase of the HR of a cardiac patient while training at home.

The model presented in this paper does not aim at replacing the well-known, usual CR programs, but is attempting to provide an alternative for patients who do not have the necessary means or time of doing CR programs in clinical environments or hospitals. This alternative should enable patients to adapt their lifestyle with little effort and in safe and monitored conditions.

Our system was developed for addressing the following research questions: 1) Is it possible to integrate multiple levels of medical expertise into smart devices for serving cardiac patients?, and 2) Can smartwatches and smartphones be alternative tools that will meet the needs of cardiac patients engaged in the second and third CR phases?.

The rest of the paper is organized as follows: Section II discusses a series of risk factors and challenges that have to be addressed for home-based CR programs, Section III reviews related work, in Section [IV] we present and describe the architecture of the entire system, including the applications for the smartwatch, the smartphone and the server, in Section $\mathrm{V}$ we present the results for testing and validating our system, and, in Sections VI and VII we conclude and we show what are the foreseen next steps in our ongoing work.

\section{RISK FACTORS AND CHALLENGES FOR HOME-BASED EXERCISE TRAINING}

When doing home-based exercises in a non-ECG environment, i.e., at home, during phase- 2 and phase- 3 of the CR program, cardiac patients are exposed to a series of risks, which may affect their safety. These risks include training at high intensity, outside the recommended limits, overtraining or sudden increases or/and decreases of HR, known as arrhythmias or abnormal heart rhythms. Challenges for the CR program include establishing an adequate training program and training limits, personalised for each patient, defining complex profiles for each patient and handling emergencies in an appropriated way.

\section{A. High-interval training versus moderate training}

Prescribing safe exercises is not an easy task. There are many guidelines and recommendations for exercises for cardiac patients like the European Society of Cardiology's guidelines [20], American College of Sports Medicine's (ACSM) guide [21] or the recommendations of the European Association for Cardiovascular Prevention and Rehabilitation
(EACPR) [22]. For example, for aerobic training, in [23], the authors recommend as intensity of the exercise $40-70 \%$ heart rate reserve (HRR) or 50-80\% maximal $\mathrm{HR}$, as frequency 3-7 days per week and as duration 20-60 minutes per session or multiple 10-min sessions. However, it is hard to determine the optimal exercise program in terms of intensity and volume [24]. There is an ongoing discussion if training in higher intensity zones substantially improves the patient's $V O_{2 p e a k}$ [25] and the exercise capacity for coronary artery disease (CAD) patients [26]. Some studies have indicated, in the case of vigorous physical activity, that the risk of sudden cardiac death [27] or myocardial infarction [28] increases.

\section{B. Overtraining}

Overtraining appears when a patient exceeds the recommended training frequency, duration, intensity or all of them. Training more often has been shown to improve not only the peak oxygen consumption $\left(\mathrm{VO}_{2 p e a k}\right)$, but also the ventilatory anaerobic threshold (VAT) and quality of life (QoL) [29]. But exceeding a certain limit of training sessions per week has minor effects on a person's physical condition [30] and can even become dangerous. The same applies to the duration of a training session. Some experts consider overtraining more risky than not training at all.

\section{Sudden changes of the HR}

Patients with cardiac arrhythmias or with high risk of cardiac arrhythmias need to be closely monitored when training. Arrhythmias, or irregular heartbeats, may be completely harmless or life-threatening. A home-based CR system should be able to detect when a patient have an abnormal increase or decrease in the HR while exercising, as indicated in Figure 3 Normally, irregular heartbeats are detected by ECG but evidence of detecting arrhythmias with a finger pulse sensor, which is also an optical sensor, was shown in [31].

\section{Low engagement level}

Inadequate training stimulation, the lack of immediate results and the long duration of training programs are only a couple of reasons for decreasing the patients' motivation and engagement in CR programs. Major beneficial effects can be seen only after longer periods of time [32] and this requires complex solutions, not only a monitoring device which shows some training limits. One solution, proposed in this study, is to organise patients into communities, based on similar characteristics and profiles, and to inform each patient about how the other patients in his/her community are performing.

\section{RELATED WORK}

Taking into consideration the focus of our research, related work can be divided into three main categories: i) ehealth remote monitoring systems or health tele-monitoring, ii) wrist-worn devices or smartwatches used for cardiac rehabilitation and iii) Proactive Systems. 


\section{A. Remote Monitoring Systems for Cardiac Rehabilitation}

The increasing need and the high significance of remote monitoring systems for post cardiac patients has been emphasized in numerous studies [33][34][35][36]. A shift can be noticed in the CR technology, from self-monitoring, selfawareness and self-determination [35] towards automated monitoring [34], automated feedback systems [33] and realtime data processing [36].

In [33], the authors proposed a mobile solution for a homebased cardiac rehabilitation program, including a server-side application and a web portal for educational materials, for providing statistics or health reports and for discussion messaging. This solution was capable of automatically turning on the application on the mobile phone, of transmitting data to the server without the user intervention and provided an automatic data synchronization of new parameters, without changing the application. However, the patients still had to provide health parameters manually to the application, including the heart rate, exercising hours, sleeping hours, etc. The system was able to provide motivational short message service (SMS), video and relaxation audio based on the received data. During the exercise sessions, patients were guided through traffic light indications. The patient's health condition was evaluated by transforming different activities like walking or cycling into MET-hours and not by continuously measuring the heart rate. A more advanced system was proposed by Kyriacou et al. (2011), where the system is supporting the recording and transmission of health parameters such as ECG, heart rate, blood pressure, oxygen saturation and others, directly from the measuring devices [34]. Despite of the advanced technological support, the data aggregation is used only for telemonitoring, and, other functionalities such as sending personalized alarms or messages according to each patient's condition or establishing the risks zones of each patient in function of his/her medical parameters and/or past training sessions, are not included.

\section{B. Wrist-worn devices for Cardiac Rehabilitation}

Monitoring continuously health parameters like the heart rate is known to have an important impact in CR programs. In clinical environment and hospitals heart rate measurements are done using an electrocardiogram (ECG). But, outside hospitals, the use of an ECG apparatus is quite limited because of the high costs and remote houses of the patients. With the current technology on the market it is now possible to record the heart rate using smartwatches that are equipped with an optical heart rate sensor. In [37], the results of a performance evaluation on the optical heart rate sensors of an LG smartwatch showed that it is reasonably accurate to measure the heart rate via a wrist-worn device. Another study on remote monitoring, using a wrist-worn device, indicated that wristworn devices can be used in home telecare, telemedicine and for detecting emergencies [38].

\section{Proactive Systems}

For this study, Proactive Computing [17] represents the key technology for making the link between all the hardware devices, the patients and the medical experts. In the past years, Proactive Systems were used for reducing the user's unnecessary intervention by providing automated actions, e.g. a service for detecting meetings and turning off the sounds of a smartphone during the meetings [39], or for reacting in case of a foreseen event or the lack of such an event, e.g. assignment submission inside a learning management system (LMS) [40]. Our implementation of Proactive Systems [41] consists of a rule-based system that works with Proactive Scenarios, representing a set of rules in which human knowledge is translated from natural language into a programming language. The rules can contain system actions, conditions, checks, database operations and other specific operations. For example, multiple Proactive Scenarios were defined in [42] for settingup communities of practice inside LMSs: one Scenario was used for creating the communities, one Scenario for promoting and maintaining the interaction and involvement inside the groups and another Scenario for closing inactive groups.

\section{THE ARCHITECTURE OF THE PROACTIVE E-HEALTH SYSTEM}

The architecture of our e-health system consists of several components: (i) the smartwatch application, (ii) the smartphone application and (iii) the server-side applications. Each component is designed to add more functionalities according to its computing capacity. Each application can work on its own and is not dependent on the other ones. More precisely, if the application on the smartphone cannot connect to the server it will continue to work as a standalone application and will analyse the data coming from the application on the smartwatch. The same case applies if the smartwatch application loses its connection to the smartphone application and cannot transmit the data coming from the sensors. It will have limited processing capacity but it will still be able to measure and display different parameters like the duration of the training, the recommended training limits and the current HR.

\section{A. The Prototype Application on the Smartwatch}

Commercial smartwatches like the Gear S2 from Samsung offer programmers the possibility of creating applications which have access to a variety of sensors on the smartwatch such as the accelerometer, gyroscope, heart rate monitor, barometer or ambient light. In order to track the activity and measure the HR of the patients involved in the CR program, a new prototype application was created for this study. The application is able to monitor and display continuously the heart rate using the watch's PPG sensor, i.e., the optical sensor, to display messages and notifications on the screen, to vibrate in case of alerts and notifications, to send raw data to the smartphone and to receive information from the smartphone via a Bluetooth connection. 


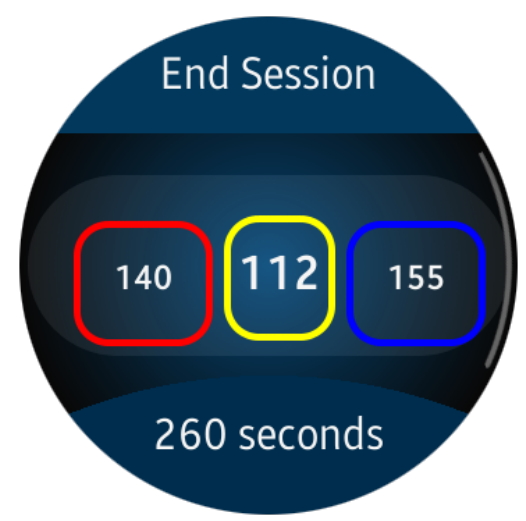

(a) The main screen of the application during ET

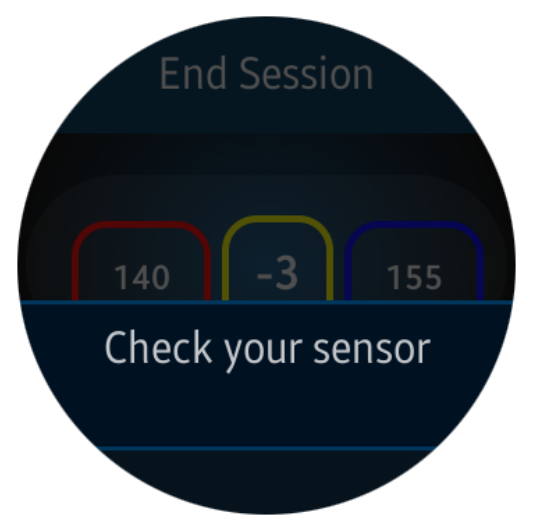

(b) A notification when the sensor does not detect a valid $\mathrm{HR}$ value

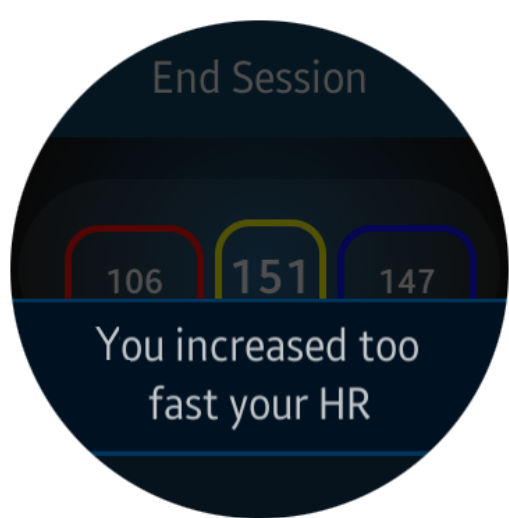

(c) Alerting the user

Fig. 1. The GUI of the application on the smartwatch Gear S2 from Samsung.

When the user starts the application, by pressing on the screen of the smartwatch on the application's icon, a new session starts to be recorded. The values of the heart rate are written to 2 files, i.e., slice.txt and session.txt, which are stored locally on the internal memory of the smartwatch. New data is written into slice.txt each 10 seconds and is sent to the smartphone for processing. The session.txt file is recording the heart rate during the entire training session and, at the end of the session, is sent to the smartphone for further processing. The need of storing data into 2 different files is motivated by the case when the smartwatch loses its connection with the smartphone and cannot send the slice.txt. In this case the corresponding data will be stored in the session.txt file and will be sent at the end of the session. The application is designed to continue running in the background during the training session, even if the user does not interact with it for a relatively long period of time. If the user does not close the application, it will close automatically if the HR is equal to -3 for more than 30 seconds. The value -3 is obtained from the optical sensor in case the UV light does not encounter any object in front of the sensor, meaning that the smartwatch may have been taken off the hand of the patient. Otherwise it displays 0 in case it detects an object but it cannot retrieve a proper HR value.

The application consists of one main screen, as presented in Figure 1a, where the current HR is shown in the centre of the screen, together with the time elapsed for the current training session and with the recommended training limits. On top of the main screen several notifications can be displayed, as seen in Figure $1 \mathrm{~b}$ and Figure $1 \mathrm{c}$. For example, in case a patient starts his/her training session and the smartwatch cannot detect the HR, a notification with the text message 'Check your sensor' will be displayed on the screen, together with a vibrating alarm. Another example, in case the patient increases the intensity of his/her training too fast, a vibrating alarm will be triggered and a notification displayed with the message 'You increased too fast your HR'. These alarms or notifications are programmed to stay on the display of the smartwatch for 5 seconds, afterwards they disappear from the screen. The application is meant to show only one notification at the time.

The interaction of the patient with the screen of the smartwatch was reduced to a minimum because the patient should be more focused on exercising correctly than on interacting with the application. Nevertheless, when a training session is completed, patients can check its details of their training session, together with other information, on the application on their smartphones.

\section{B. The Prototype Application on the Smartphone}

In order to acquire raw data from the watch and to process it, a prototype application for Android-based mobile phones was created. The application is receiving data each 10 seconds, via Bluetooth, from the smartwatch application when an exercise session is started via a text file called slice.txt and, at the end of the exercise session, via a text file called session.txt. If a slice is missed, the training session can be reconstructed based on the session file. The other way around works as well, meaning that if the session text file is not received, the session's data can be reconstructed from the slice text files.

The application has multiple screens where the patient can consult the details of his/her last training session, see a list with previous sessions and see statistics about themselves and their communities. In Figure $2 \mathrm{a}$ the main screen of the application, in the upper side of the screen, the patient can check if he/she respected the recommendations established before, at the hospital, in terms of duration, frequency and intensity of a training session. In the lower part of the screen, coaching messages are displayed to the user for guiding or advising purposes. It will either tell the patients to improve their training sessions or to continue, in case the exercise session was according to the medical guidelines. The second screen of the application contains a list of previous sessions, presented in Figure 2b, and, when clicked on one of them, a new screen which contains the summary of the clicked session will open. And, finally, Figure 2c shows the last screen which contains multiple facts and statistics, such as the number of people in 


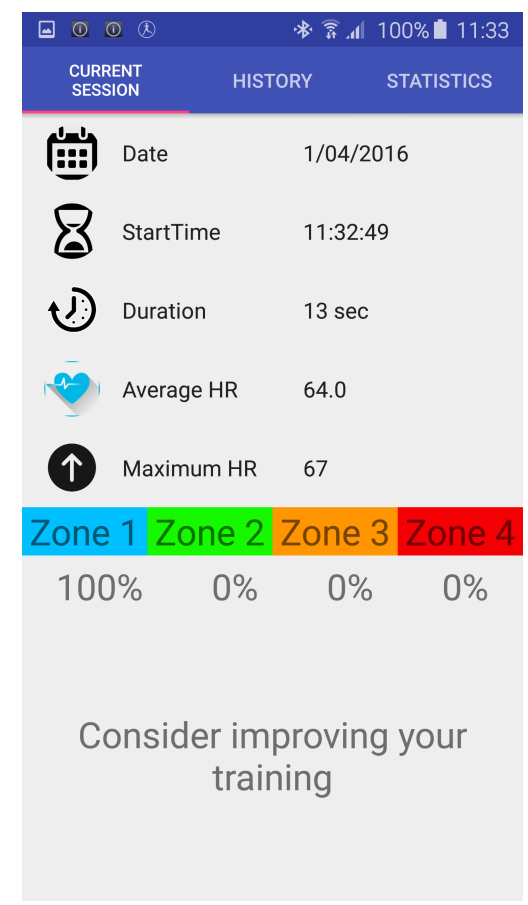

(a) Screen 1 - The summary of the last training session

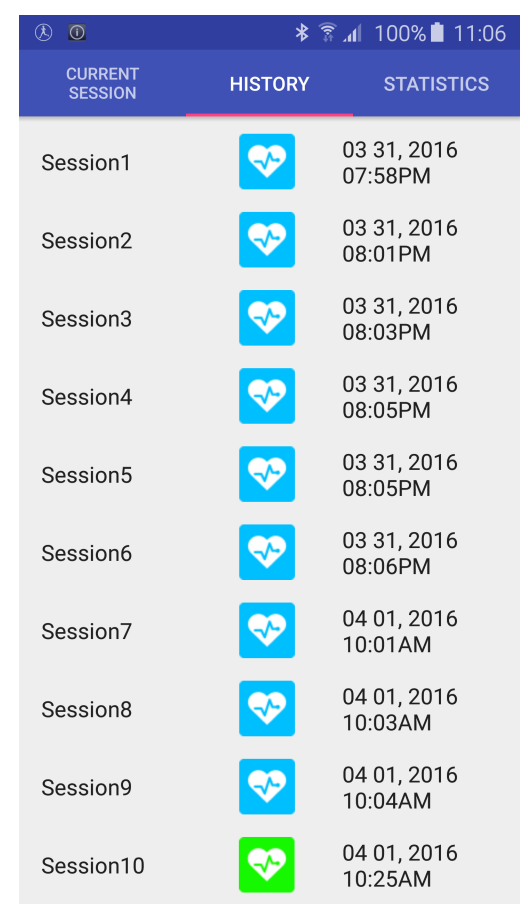

(b) Screen2 - A list with previous training sessions

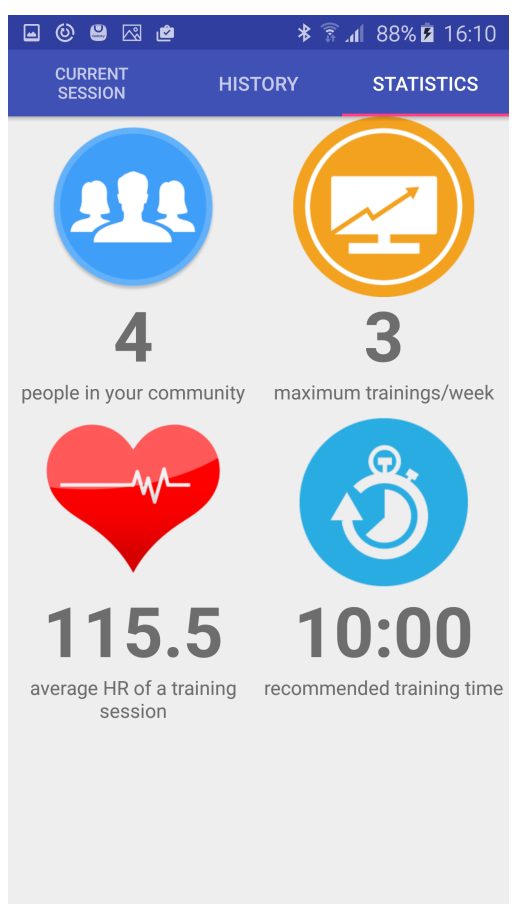

(c) Screen3 - Statistics about the patient and his/her community

Fig. 2. The GUI of the smartphone application

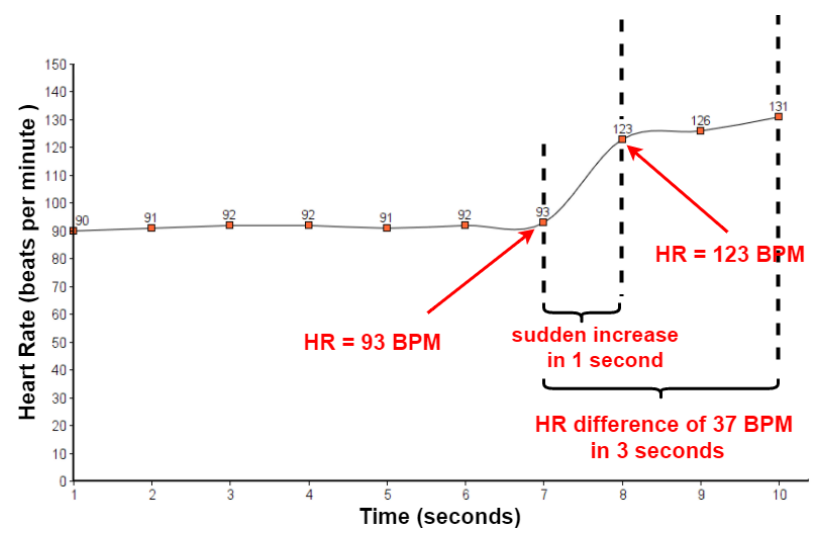

Fig. 3. Sudden increase of the HR during a training session

the patient's community which are also participating in the same CR program, the average HR of all the previous training sessions, the maximum training times of a single patient from the community and the hour recommended to begin training. Communities are created on the server side for tackling the problem of low patient motivation and engagement discussed in Section II-D. This can create extra motivation factors which will increase the participation of patients in their $\mathrm{CR}$ programs. Note that this last screen currently contains only a basic set of statistics and facts and will be easily extended in the future.

The most important part is how the application works and how it is designed to analyse the data. The application works on top of the Proactive Engine (PE) for mobile devices, which is a middleware architecture used for transforming reactive mobile applications into proactive applications. The PE works with Proactive Scenarios that are processed and executed periodically, e.g., each 3 seconds. Proactive Scenarios represent a method for implementing human knowledge or expert knowledge into an application. More specifically, like other expert systems [43] [44], they work with a knowledge base and with rules, which are then processed and executed by an inference engine. For example, a Proactive Scenario can take care of detecting if a patient suddenly increases or decreases his/her HR during a training session. If the patient is at 110 beats per minute (BPM) and is arriving at 150 BPM in less than 5 seconds and it stays high or continues to increase, as shown in the Figure 3, then, the cardiac patient can have a serious problem. Or, it can be caused by the sensor, which can misread the correct value of the HR, or it can be caused by the communication channel between the smartphone and the smartwatch, which can lose data. The complexity of this problem is future described in [45] or [46]. However, the specifications of the smartwatch and its operating system do not make it a candidate for supporting middleware architectures, like a PE, and for computing complex rules, while the smartphone does.

Several Proactive Scenarios were developed for the application on the smartphone for handling part of the challenges described in Section II The Proactive Scenarios are divided into two main categories on the smartphone: local scenarios and global scenarios. Local scenarios have access to enough information to take decisions and execute actions locally, 


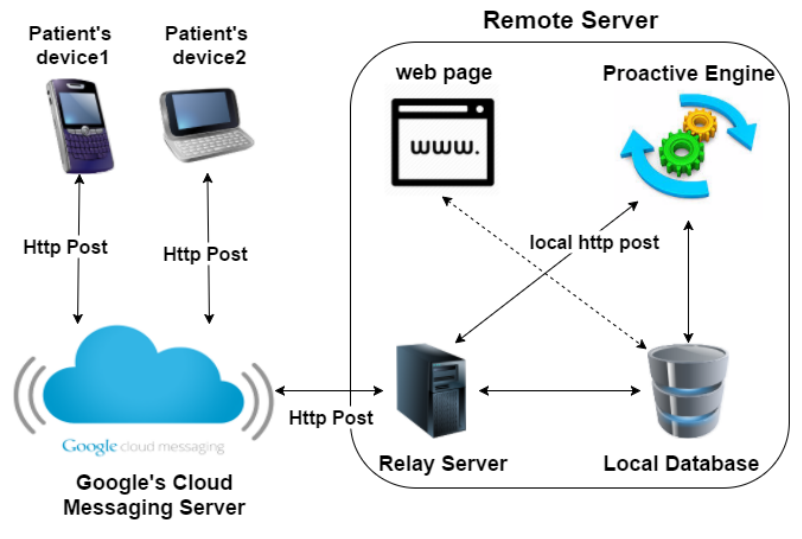

Fig. 4. The server-side architecture

while global scenarios imply several PEs working together for solving a bigger puzzle [47]. Proactive scenarios have multiple activation procedures, varying from the occurrence or lack of simple events to sequences of events and can perform multiple complex actions, e.g., like alerting a patient when overtraining.

The smartphone application includes 4 local scenarios and 2 global scenarios. Local Scenario 1 was developed for detecting sudden HR changes during exercise sessions, Local Scenario 2 for checking the intensity of the training sessions, Local Scenario 3 for establishing training zones and how much does the patient train in each zone and Local Scenario 4 for creating coaching messages for the patient. The two global scenarios, i.e., Global Scenario 1 and Global Scenario 2, contain rules on both sides, on the smartphone's side and on the server's side. The first one was developed for dynamically adjusting the training limits of a patient, while the second one was created to handle the communities of patients. A concrete example of the implementation of Global Scenario 2 is given in Section IV-E The Proactive Scenarios used for the study were discussed together with doctors and medical specialists working at the 2 local hospitals.

\section{The Server-side Layer}

As depicted in Figure 4, the architecture of the server-side layer is divided into several parts: the PE, the Relay Server, the local database and a website. While the mobile application is in charge of handling local data related to each patient, the server-side applications are responsible of collecting, keeping track and analysing all the processed data coming from all the mobile phones. More specifically, the Relay Server is used for receiving and sending messages from and to mobile phones. The website contains a personalised profile for each patient and is in charge of displaying statistics related to his/her previous exercise sessions. A doctor can access the results of the exercise session of any patient he/she is in charge of, he/she can compare it with the other patients' sessions and can update the profile of each patient, thus increasing or decreasing the safety limits of a patient's heart rate during training. And, finally, the PE, a powerful Java rule-based engine, capable of running Proactive Rules which compose Proactive Scenarios, is in charge of taking actions based on the information it receives. A performance test for a PE running on a server with small computing capacity shows that it was able to execute 1000 Proactive Rules in 220 milliseconds compared with 1295 milliseconds on a smartphone [48].

To take advantage of the global knowledge available on the server, several local and global Proactive Scenarios were developed. Local Scenario $S 1$ is in charge of periodically generating medical reports containing the evolution of a patient in the CR program from the beginning until the time of checking. Information is available regarding the number of training per week, the intensity and the duration of trainings, the time spent in each training zone, for each session, the date and the starting hours of the exercise sessions. The reports generate a PDF file which is available to the specialist or doctor via the website. This scenario does not wait for the doctor's explicit command but is building and sending the report automatically. The other scenarios are global, one for the dynamic profile of the patients, one for handling the communities and another one for providing extended medical coaching. For instance, Global Scenario GS2 checks which patients are not exercising according to their CR program and sends messages to encourage them. These messages can contain statistics about their communities or about certain patients in their communities. Global Scenario GS3 performs one analysis on how much each patient stayed in each training zone, e.g., how much time they were training at $55 \%$ of their maximum HR, compares it with previous sessions of the patient and decides if the patient needs to increase the training time spent in one training zone or decrease this time, in case the training zone was too high, e.g., 5 minutes at $70 \%$ of the maximum HR. And Global Scenario GS4 is in charge of sending by email periodical reports, containing patient relevant information regarding the training frequency, intensity and duration, to the specialists in charge of the patients.

Communities of patients with different characteristics are created and managed on the server side via the web interface by the medical experts. The possibility of automatically generating the communities, based on the profile of the patients, exists and can be done with the help of extra Proactive Scenarios. However, as it was not the main focus of this study, it was left to be manually handled and supervised by the medical experts. So, for example, with the current settings, an expert could decide to create a community of male patients with the same cardiac affection, aged between 50 and 55, if there were at least 3 patients with these characteristics. The supervision of the communities is done by the medical experts via the web interface and via the periodical reports, which contain more specific data about each patient compared to his/her community.

\section{Multiple Levels of Feedback and Monitoring}

Real-time feedback is a very important aspect when working with patients with heart conditions. A simple alert can warn a cardiac patient if he/she is doing something wrong and this can avoid accidents, even saving his/her life in the given 


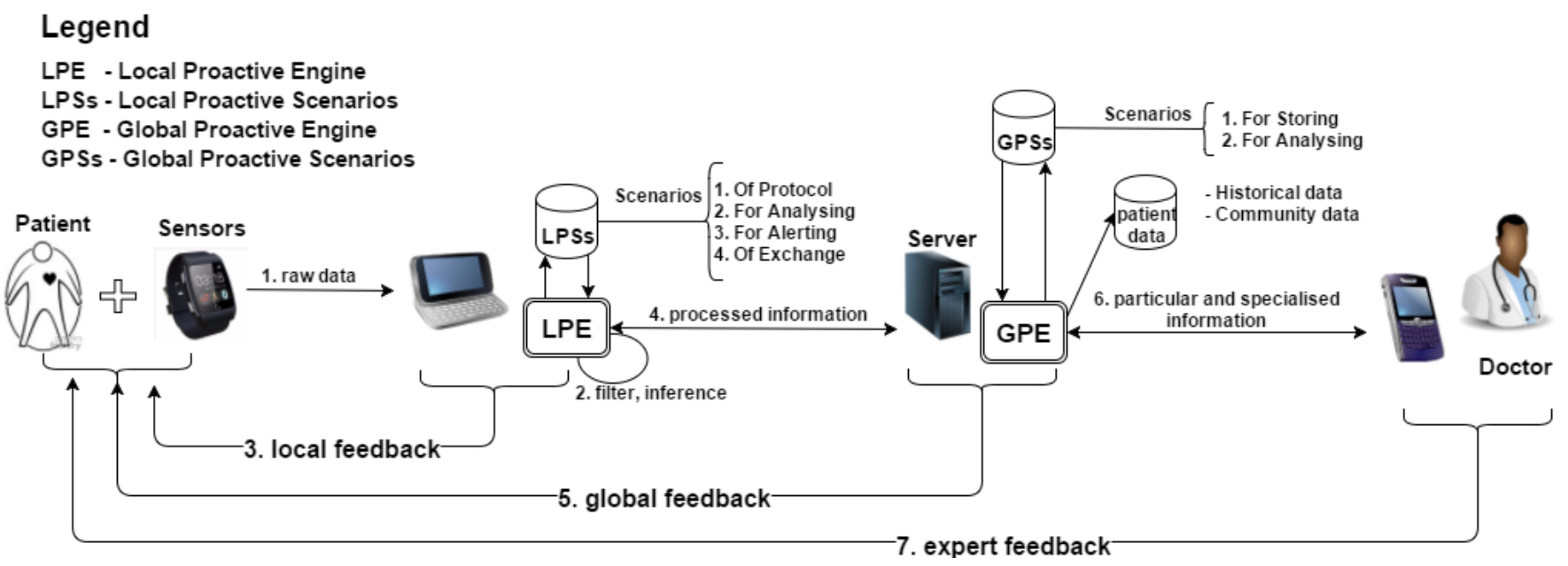

Fig. 5. The architecture of the Proactive E-Health System with multiple levels of monitoring and expertise

circumstances of cardiac rehabilitation. Different types of expertise are available to patients when doing the cardiac rehabilitation exercises, as shown in Figure 5 . First, a local level of expertise is embedded into the smartwatch and the mobile phone thus providing local feedback, the second level, more extensive and based on a broader context, comes from the server and the third level comes directly from the medical experts and their personal devices.

1) Local level of expertise: The applications on the smartwatch and on the mobile phone are able to acquire data, analyse it locally and provide patients with immediate feedback. On the mobile phone, the prototype application is performing more complex analyses with the help of Proactive Rules, which contain a set of minimum medical knowledge. This first loop between the patient, the sensors of the smartwatch, its screen and the mobile phone offers the necessary local medical expertise.

2) Global level of expertise: At a more global level, on the server side, data is gathered from all the smartphones of all the patients. At this stage, a more powerful PE in terms of computing capacity is available for analysing and aggregating information from all the smartphones. As described in Section IV-C the computing capabilities of a similar PE are described in [48]. The full data set of the patients is stored on the server, thus offering the possibility of analysing historical data and of data mining techniques. Progressive monitoring is also available on the server's side, meaning that monitoring parameters can change after more information about each patient is gathered.

3) The specialist's direct advice: This level of feedback was designed to keep the doctors in the loop even when they are not at the hospital. They can be informed on their personal devices, e.g., mobile phones, if patients were up to date with their cardiac rehabilitation program, they can receive notifications about certain patients with more severe forms of heart conditions and/or they can be alerted in case a patient had problems during his/her training sessions and did not stop according to the indications of the local Proactive System. In this last case, the doctor can immediately call the patient to discuss with him/her, to see what happened exactly and to take measures to prevent unpleasant heart event.

\section{E. Dynamic Patient Profiles}

As discussed in Section [I] establishing training limits for each patient is a big challenge, as training outside the correct limits can be dangerous, even fatal. A post event symptomlimited graded exercise test (GXT) at the hospital, before the patient's discharge, has to be done for determining the exercise capacity of each patient. More precisely, it measures the peak HR (measuredH $R_{\max }$ ) of a patient and determines the anaerobic threshold (AT), which is the point where energy production is supplemented by anaerobic mechanisms [49]. Another way of defining it is the exertion level between anaerobic and aerobic training. AT is very important because of the differences in physiological responses of patients during training below and above AT. Cardiac patients have lower values for AT because of their conditions.

Available applications do not offer personalised training boundaries, i.e., target heart rate zones, for cardiac patient. For example, Polar gives the users the possibility of manually setting the training limits or of performing a test session with the OwnZone program set to determine the limits of each user [50]. Training zones are determined by the sensors on the chest strap connected to the watch or, if the heart rate rose too fast during the measurements, on age-based algorithms. But, if health changes appear for certain patients, e.g., they start to take medication, the limits and training zones are not updated accordingly.

We propose a solution, based on Proactive Computing, where the profile of each patient is calculated dynamically with the help of a Global Proactive Scenario (GPaS). When the application is installed it comes with limits by default calculated as if the patient would not have a stress test. Then, the server pushes to the patient's smartphone necessary infor- 
mation for creating his/her training zones and HR limits such as age, anaerobic threshold if existing, maximum measured HR or if the patient is under treatment or not. Afterwards, these limits are automatically pushed to the smartwatch of the patient. The medical specialist, who controls the profile of each patient remotely via a website, can change certain parameters and so, the profile is then calculated again according to the new parameters. For example, initially, a profile does not have an anaerobic threshold value included neither a maximum measured HR and so, his/her training limits will be calculated based on his/her age. As soon as the specialist introduces the AT's value, the limits are adjusted automatically, so, when the patient will start his/her next exercise session, the new limits will be displayed on the smartwatch.

Several cases can be distinguished when establishing training limits for cardiac patients, as the one for endurance training, where the training interval is equal to $70 \%-85 \%$ of the measured $H R_{\max }$ [51]. For this study, target heart rate (THR) zones were established for performing aerobic exercises. In case the measuredHR $R_{\max }$ of a patient is known and the anaerobic threshold is not, and the patient is under treatment, then the recommended THR interval is between $65 \%$ - $90 \%$ of the measuredH $R_{\max }$. Moreover, if the patient is not under treatment but values are known for his/her measured $H R_{\max }$ and AT, the THR interval becomes $[A T-10 \mathrm{bpm}]$ for the lower limit and $[A T+5 \mathrm{bpm}]$ for the upper limit. On the other hand, if the patient does not have an AT neither a measured $H R_{\max }$ and is under medication, then the THR interval is between 55\% and $77 \%$ of the theoreticalH $R_{\max }$, which is determined by the well known formula (220 - age) [52]. Otherwise, if he/she is not under medication, the lower limit and upper limits are set to $65 \%$, respectively $90 \%$ of the theoretical $H R_{\max }$.

\section{System Testing and Evaluation}

In order to test and evaluate our system in a real world scenario, an experimental study was performed. The study involved collecting data from 5 men from our team, at the local university, for a duration of 8 weeks. The low number of people and the fact of not using real cardiac patients resulted from the difficulties of obtaining an authorization for working with real patients involved in a cardiac rehabilitation program, which takes minimum 1 year in our country.

The participants were instructed, at the beginning of the study, to register training sessions when performing physical activities during the day. Their age ranged from 21 to 48 years old, being part of the same community. They did not take any medication during the period of the experiments. So, the recommended exercise training limits for each participant was calculated using the formula discussed in the Section IV-E i.e., $65-90 \%$ of the theoreticalH $R_{\max }$, (220 - age). Each person participating in the experiments was given a Samsung Galaxy S6 smartphone and a Gear S2 martwatch from Samsung. A unique email address was assigned to each of the 5 participants. Each smartphone was equipped with a Proactive Engine and with the prototype application running on top of it. A special Bluetooth channel for exchanging data

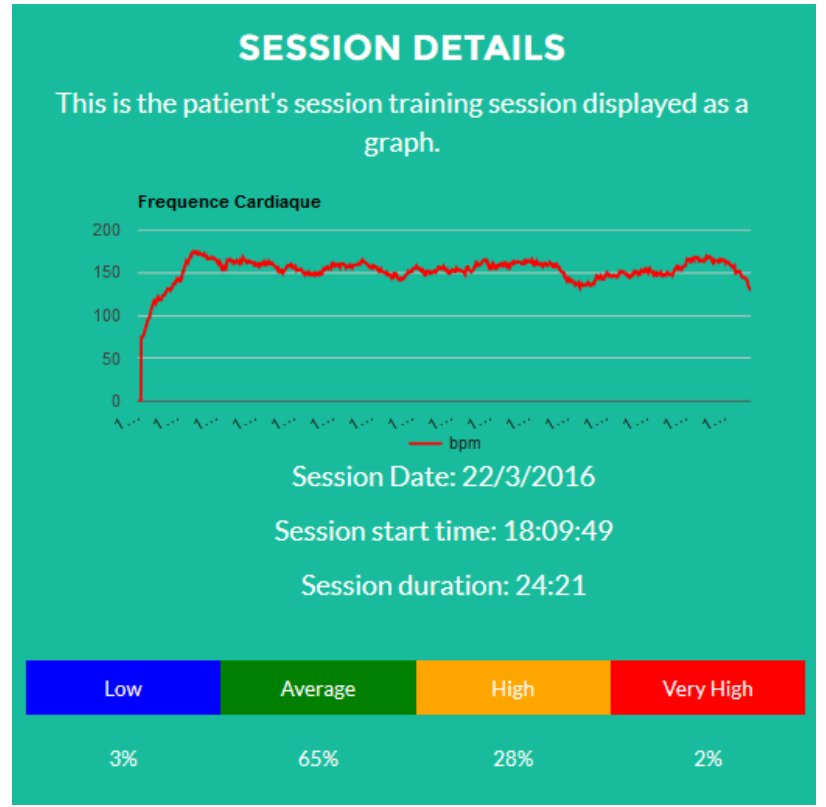

Fig. 6. A 25 minutes training session which was registered on the server-side without any errors

was defined between the devices for not interfering with other Bluetooth enabled devices.

A total of 90 valid training sessions were registered in the server's database. A training session was considered valid if it contained values bigger than 10 BPM. The smartwatch was able to register continuously the HR during entire training sessions, as the one shown in Figure 6, without any loss of data. Figure 6 shows one example of a usual training session, starting from $0 \mathrm{bpm}$, when opening the application, moving up to around $50 \mathrm{bpm}$, when a correct HR value was initially detected, and then continuing to increase up to a value in between $150 \mathrm{bpm}$ and $165 \mathrm{bpm}$. This intensity interval was then kept for the rest of the training session.

Changes on the profiles were performed several times and limits on the smartwatches were adjusted either during training sessions or later on, when starting a new training session, thus checking the Proactive Scenarios regarding the patient profiles on the server and on the smartphone. The participants reported multiple times the appearance of alarms and notifications when they were overpassing the recommended training limits, when they were not training at the recommended frequency and when the sensor could not detect a HR value. Coaching messages were displayed after each session and were kept visible until the start of new session, thus validating the local scenarios on the smartphones. A few cases were detected of smaller sessions containing non-valid HR values, followed by longer training sessions with valid HR values. This fact occurred when the sensor of the smartwatch was not initially placed correctly and when the smartwatch application was restarted. 


\section{CONCLUSION}

The main goal of this study was achieved by providing a proactive monitoring and coaching system that will allow patients, participating in a home-based CR program, to train in safe limits, to improve their health condition and to develop a long term habit of exercising, while keeping the medical experts in the supervising role. Also, the model makes it possible to take cardiac rehabilitation medical knowledge and to integrate it, with the help of Proactive Computing, on wearable devices, on mobile phones and on remote servers, thus validating the first research question. The tests showed promising results in favour of using wearable devices as suitable tools for registering entire training sessions, mobiles for analysing single patient-based data and servers for aggregating data from entire communities of patients, hence, verifying the second research question. This study also addresses a topic which is becoming increasingly popular, i.e., the utilisation of wearable sensors in e-Health rehabilitation applications.

Our model complements the traditional cardiac rehabilitation programs that take place in specialized clinics, while offering patients an alternative for their rehabilitation training, while maintaining the connection patient-doctor. At the same time, by providing patients with a proactive automated wearable and mobile application, we attempted to reduce the patient's interaction with the system to a minimum, thus, allowing him/her to focus more on his/her training sessions. The bigger goal of this model is intended to expand, support and increase the utilization of cardiac rehabilitation programs.

Finally, wearable devices, mobile phones and web technology, combined together, present a huge potential for homebased cardiac rehabilitation programs. With the help of the new generation of sensors available on the market, the model presented in this paper could be extended for monitoring, guiding and helping patients with different health problems.

\section{Future STEPS}

The next step is to use our system in a CR program with real cardiac patients, in collaboration with a team of medical experts from the local hospitals. During this next phase, quantitative and qualitative data will be collected for finding a correlation between home training with the help of the proposed proactive system and the patient's motivation and determination. The idea is to split patients into 2 groups with similar characteristics in terms of age, gender, cardiac affection: one monitored target group with multiple communities of patients, where our system automatically collects data, guides, helps and alerts the patients, and another monitored group, where the patients will have to manually send their training summary after each training session to the clinic. Moreover, specialised tests will be done at the local hospitals or clinics, in the beginning and at the end of the study, for determining if there will be any major differences in the patients' health conditions, e.g., an increase in the $\mathrm{VO}_{2 \max }$ capacity of the patients.

\section{REFERENCES}

[1] D. Mozaffarian, E. J. Benjamin, A. S. Go, D. K. Arnett, M. J. Blaha, M. Cushman, S. de Ferranti, J.-P. Despres, H. J. Fullerton, V. J. Howard et al., "Heart disease and stroke statistics-2015 update: a report from the american heart association." Circulation, vol. 131, no. 4, p. e29, 2015.

[2] World Health Organization, "Rehabilitation after cardiovascular diseases, with special emphasis on developing countries: report of a who expert committee," 1993.

[3] H. Gohlke and C. Gohlke-Barwolf, "Cardiac rehabilitation," European heart journal, vol. 19, no. 7, pp. 1004-1010, 1998.

[4] The British Association for Cardiovascular Prevention and Rehabilitation. (2012) The BACPR Standards and Core Components for Cardiovascular Disease Prevention and Rehabilitation 2012 (2nd Edition). Online; accessed 13-December-2015]. [Online]. Available: http://www.bacpr.com/resources/15E_BACPR_Standards_FINAL.pdf

[5] B. S. Heran, J. Chen, S. Ebrahim, T. Moxham, N. Oldridge, K. Rees, D. R. Thompson, and R. S. Taylor, "Exercise-based cardiac rehabilitation for coronary heart disease," Cochrane Database Syst Rev, vol. 7, no. 7, 2011.

[6] D. Thompson and D. De Bono, "How valuable is cardiac rehabilitation and who should get it?" Heart, vol. 82, no. 5, pp. 545-546, 1999.

[7] H. Antonakoudis, K. Kifnidis, A. Andreadis, E. Fluda, Z. Konti, N. Papagianis, H. Stamou, E. Anastasopoulou, G. Antonakoudis, and L. Poulimenos, "Cardiac rehabilitation effects on quality of life in patients after acute myocardial infarction," Hippokratia, vol. 10, no. 4, p. 176, 2006.

[8] R. Arena, M. Williams, D. E. Forman, L. P. Cahalin, L. Coke, J. Myers, L. Hamm, P. Kris-Etherton, R. Humphrey, V. Bittner et al., "Increasing referral and participation rates to outpatient cardiac rehabilitation: The valuable role of healthcare professionals in the inpatient and home health settings a science advisory from the american heart association," Circulation, vol. 125, no. 10, pp. 1321-1329, 2012.

[9] P. D. Thompson, B. A. Franklin, G. J. Balady, S. N. Blair, D. Corrado, N. M. Estes, J. E. Fulton, N. F. Gordon, W. L. Haskell, M. S. Link et al., "Exercise and acute cardiovascular events placing the risks into perspective: a scientific statement from the american heart association council on nutrition, physical activity, and metabolism and the council on clinical cardiology," Circulation, vol. 115, no. 17, pp. 2358-2368, 2007.

[10] D. R. Thompson and R. J. Lewin, "Management of the post-myocardial infarction patient: rehabilitation and cardiac neurosis," Heart, vol. 84, no. 1, pp. 101-105, 2000.

[11] S. Patel, H. Park, P. Bonato, L. Chan, and M. Rodgers, "A review of wearable sensors and systems with application in rehabilitation," Journal of NeuroEngineering and Rehabilitation, vol. 9, no. 1, pp. 1-17, 2012. [Online]. Available: http://dx.doi.org/10.1186/1743-0003-9-21

[12] J. R. Windmiller and J. Wang, "Wearable electrochemical sensors and biosensors: A review," Electroanalysis, vol. 25, no. 1, pp. 29-46, 2013. [Online]. Available: http://dx.doi.org/10.1002/elan.201200349

[13] U. Anliker, J. A. Ward, P. Lukowicz, G. Tröster, F. Dolveck, M. Baer F. Keita, E. B. Schenker, F. Catarsi, L. Coluccini et al., "Amon: a wearable multiparameter medical monitoring and alert system," Information Technology in Biomedicine, IEEE Transactions on, vol. 8, no. 4, pp. 415-427, 2004.

[14] M. Bächlin, M. Plotnik, D. Roggen, I. Maidan, J. M. Hausdorff, N. Giladi, and G. Tröster, "Wearable assistant for parkinsons disease patients with the freezing of gait symptom," Information Technology in Biomedicine, IEEE Transactions on, vol. 14, no. 2, pp. 436-446, 2010.

[15] J. Hernandez, D. McDuff, and R. W. Picard, "Biowatch: estimation of heart and breathing rates from wrist motions," in Proceedings of the 9th International Conference on Pervasive Computing Technologies for Healthcare. ICST (Institute for Computer Sciences, Social-Informatics and Telecommunications Engineering), 2015, pp. 169-176.

[16] J. Allen, "Photoplethysmography and its application in clinical physiological measurement," Physiological measurement, vol. 28, no. 3, p. R1, 2007.

[17] D. Tennenhouse, "Proactive computing," Communications of the ACM, vol. 43, no. 5, pp. 43-50, 2000.

[18] A. Salovaara and A. Oulasvirta, "Six modes of proactive resource management: a user-centric typology for proactive behaviors," in Proceedings of the third Nordic conference on Human-computer interaction. ACM, 2004, pp. 57-60. 
[19] D. Shirnin, S. Reis, and D. Zampunieris, "Design of proactive scenarios and rules for enhanced e-learning," in CSEDU 2012. SciTePressScience and Technology Publications, 2012, pp. 253-258.

[20] J. J. McMurray, S. Adamopoulos, S. D. Anker, A. Auricchio, M. Böhm, K. Dickstein, V. Falk, G. Filippatos, C. Fonseca, M. A. Gomez-Sanchez et al., "Esc guidelines for the diagnosis and treatment of acute and chronic heart failure 2012," European journal of heart failure, vol. 14, no. 8, pp. 803-869, 2012.

[21] A. C. of Sports Medicine et al., ACSM's guidelines for exercise testing and prescription. Lippincott Williams \& Wilkins, 2013.

[22] M. F. Piepoli, V. Conraads, U. Corra, K. Dickstein, D. P. Francis, T. Jaarsma, J. McMurray, B. Pieske, E. Piotrowicz, J.-P. Schmid et al., "Exercise training in heart failure: from theory to practice. a consensus document of the heart failure association and the european association for cardiovascular prevention and rehabilitation," European journal of heart failure, vol. 13, no. 4, pp. 347-357, 2011.

[23] N. F. Gordon, M. Gulanick, F. Costa, G. Fletcher, B. A. Franklin, E. J. Roth, and T. Shephard, "Physical activity and exercise recommendations for stroke survivors an american heart association scientific statement from the council on clinical cardiology, subcommittee on exercise, cardiac rehabilitation, and prevention; the council on cardiovascular nursing; the council on nutrition, physical activity, and metabolism; and the stroke council," Stroke, vol. 35, no. 5, pp. 1230-1240, 2004.

[24] P. Kokkinos and J. Myers, "Exercise and physical activity clinical outcomes and applications," Circulation, vol. 122, no. 16, pp. 1637$1648,2010$.

[25] T. Moholdt, E. Madssen, Ø. Rognmo, and I. L. Aamot, "The higher the better? interval training intensity in coronary heart disease," Journal of Science and Medicine in Sport, vol. 17, no. 5, pp. 506-510, 2014.

[26] V. M. Conraads, N. Pattyn, C. De Maeyer, P. J. Beckers, E. Coeckelberghs, V. A. Cornelissen, J. Denollet, G. Frederix, K. Goetschalckx, V. Y. Hoymans et al., "Aerobic interval training and continuous training equally improve aerobic exercise capacity in patients with coronary artery disease: the saintex-cad study," International journal of cardiology, vol. 179, pp. 203-210, 2015.

[27] D. S. Siscovick, N. S. Weiss, R. H. Fletcher, and T. Lasky, "The incidence of primary cardiac arrest during vigorous exercise," New England Journal of Medicine, vol. 311, no. 14, pp. 874-877, 1984.

[28] S. Giri, P. D. Thompson, F. J. Kiernan, J. Clive, D. B. Fram, J. F. Mitchel, J. A. Hirst, R. G. McKay, and D. D. Waters, "Clinical and angiographic characteristics of exertion-related acute myocardial infarction," Jama, vol. 282, no. 18, pp. 1731-1736, 1999.

[29] W. Nieuwland, M. A. Berkhuysen, D. J. van Veldhuisen, J. Brügemann, M. L. Landsman, E. van Sonderen, K. Lie, H. J. Crijns, and P. Rispens, "Differential effects of high-frequency versus low-frequency exercise training in rehabilitation of patients with coronary artery disease," Journal of the American College of Cardiology, vol. 36, no. 1, pp. 202207, 2000.

[30] A. C. of Sports Medicine et al., "ACSM's guidelines for exercise testing and prescription," in Adaptations to cardiorespiratory exercise training, D. P. Swain and C. A. Brawner, Eds. Oxford: Oxford University Press, 2013, ch. 32, pp. 496-510.

[31] R. W. Kusuma, R. A. A. Abbie, and P. Musa, "Design of arrhythmia detection device based on fingertip pulse sensor," in Proceedings of Second International Conference on Electrical Systems, Technology and Information 2015 (ICESTI 2015). Springer, 2016, pp. 363-372.

[32] S. Magalhães, M. M. Ribeiro, A. Barreira, P. Fernandes, S. Torres, J. L. Gomes, and S. Viamonte, "Long-term effects of a cardiac rehabilitation program in the control of cardiovascular risk factors," Revista Portuguesa de Cardiologia (English Edition), vol. 32, no. 3, pp. 191199, 2013

[33] A. Sarela, J. Salminen, E. Koskinen, O. Kirkeby, I. Korhonen, and D. Walters, "A home-based care model for outpatient cardiac rehabilitation based on mobile technologies," in Pervasive Computing Technologies for Healthcare, 2009. PervasiveHealth 2009. 3rd International Conference on, April 2009, pp. 1-8.

[34] E. Kyriacou, P. Chimonidou, C. Pattichis, E. Lambrinou, V. Barberis, and G. Georghiou, "Post cardiac surgery home-monitoring system," in Wireless Mobile Communication and Healthcare, ser. Lecture Notes of the Institute for Computer Sciences, Social Informatics and Telecommunications Engineering, J. Lin and K. Nikita, Eds. Springer Berlin Heidelberg, 2011, vol. 55, pp. 61-68. [Online]. Available: http://dx.doi.org/10.1007/978-3-642-20865-2_9
[35] J. Maitland and M. Chalmers, "Self-monitoring, self-awareness, and self-determination in cardiac rehabilitation," in Proceedings of the SIGCHI Conference on Human Factors in Computing Systems, ser. CHI '10. New York, NY, USA: ACM, 2010, pp. 1213-1222. [Online]. Available: http://doi.acm.org/10.1145/1753326.1753508

[36] V. Gay, P. Leijdekkers, and E. Barin, "A mobile rehabilitation application for the remote monitoring of cardiac patients after a heart attack or a coronary bypass surgery," in Proceedings of the 2Nd International Conference on PErvasive Technologies Related to Assistive Environments, ser. PETRA '09. New York, NY, USA: ACM, 2009, pp. 21:1-21:7. [Online]. Available: http: //doi.acm.org/10.1145/1579114.1579135

[37] D. Phan, L. Y. Siong, P. Pathirana, and A. Seneviratne, "Smartwatch: Performance evaluation for long-term heart rate monitoring," in Bioelectronics and Bioinformatics (ISBB), 2015 International Symposium on, Oct 2015, pp. 144-147.

[38] J. M. Kang, T. Yoo, and H. C. Kim, "A wrist-worn integrated health monitoring instrument with a tele-reporting device for telemedicine and telecare," Instrumentation and Measurement, IEEE Transactions on, vol. 55 , no. 5 , pp. $1655-1661$, Oct 2006

[39] R. Dobrican, G. Neyens, and D. Zampunieris, "Silentmeet - a prototype mobile application for real-time automated group-based collaboration," in Proceedings of the 5th International Conference on Advanced Collaborative Networks, Systems and Applications (COLLA 2015), 2015, pp. 52-56.

[40] S. Reis, D. Shirnin, and D. Zampunieris, "Design of proactive scenarios and rules for enhanced e-learning," in Proceedings of the 4th International Conference on Computer Supported Education, 2012, pp. 253258.

[41] D. Zampunieris, "Implementation of a proactive learning management system," in Proceedings of" E-Learn-World Conference on E-Learning in Corporate, Government, Healthcare \& Higher Education", 2006, pp. 3145-3151.

[42] R. Dobrican and D. Zampunieris, "Supporting collaborative learning inside communities of practice through proactive computing," in $E D$ ULEARN13 Proceedings, ser. 5th International Conference on Education and New Learning Technologies. IATED, 1-3 July, 2013 2013, pp. $5824-5833$

[43] R. Ahmad, "Expert systems: Principles and programming," Scalable Computing: Practice and Experience, vol. 7, no. 4, 2001.

[44] J. C. Giarratano and G. Riley, Expert systems. PWS Publishing Co., 1998.

[45] Y. Shahar, D. Goren-Bar, D. Boaz, and G. Tahan, "Distributed, intelligent, interactive visualization and exploration of time-oriented clinical data and their abstractions," Artificial intelligence in medicine, vol. 38, no. 2, pp. 115-135, 2006.

[46] C. Combi, G. Pozzi, and R. Rossato, "Querying temporal clinical databases on granular trends," Journal of biomedical informatics, vol. 45 , no. 2, pp. 273-291, 2012

[47] R. A. Dobrican and D. Zampunieris, "A proactive approach for information sharing strategies in an environment of multiple connected ubiquitous devices," in Ubiquitous Intelligence and Computing, 2014 IEEE 11th Intl Conf on and IEEE 11th Intl Conf on and Autonomic and Trusted Computing, and IEEE 14th Intl Conf on Scalable Computing and Communications and Its Associated Workshops (UTC-ATC-ScalCom), Dec 2014, pp. 763-770.

[48] G. Neyens, R.-A. Dobrican, and D. Zampunieris, "Enhancing mobile devices with cooperative proactive computing," in Proceedings of the 5th International Conference on Advanced Collaborative Networks, Systems and Applications (COLLA 2015). IARIA, 2015, pp. 1-9.

[49] K. Wasserman, "The anaerobic threshold: definition, physiological significance and identification," 1986.

[50] Polar Electro, "How to do OwnZone determination," March 2016. [Online]. Available: http://support.polar.com/en/support/tips/How_to_ do OwnZone determination

[51] L. A. Sebastian, S. Reeder, and M. Williams, "Determining target heart rate for exercising in a cardiac rehabilitation program: A retrospective study," Journal of Cardiovascular Nursing, vol. 30, no. 2, pp. 164-171, 2015.

[52] S. M. Fox, J. P. Naughton, and W. Haskell, "Physical activity and the prevention of coronary heart disease," in Ann Clin Res, 1971, pp. 404432. 